\title{
INDIVIDUAL AND GENDER DIFFERENCES IN PERSONALITY INFLUENCE THE DIAGNOSIS OF MAJOR DEPRESSIVE DISORDER
}

\author{
Sanja Nikolic ${ }^{1}$, Ivana Perunicic Mladenovic ${ }^{1}$, Olivera Vukovic ${ }^{1,2}$, Jasmina Barišić ${ }^{3}$, \\ Dragan Švrakić $^{4}$ \& Srdjan Milovanović ${ }^{2,3}$ \\ ${ }^{1}$ Institute of Mental Health, Belgrade, Serbia \\ ${ }^{2}$ Faculty of Medicine, University of Belgrade, Belgrade, Serbia \\ ${ }^{3}$ Clinic for Psychiatry, Clinical Center of Serbia, Belgrade, Serbia \\ ${ }^{4}$ Department of Psychiatry, Washington University School of Medicine in St Louis, USA \\ received: 26.3.2019; \\ revised: 8.1.2020; \\ accepted: 16.1 .2020
}

\section{SUMMARY}

Background: In order to explore whether gender differences are present in self-reports on personality measures when all Major Depressive Disorder (MDD) participants are diagnosed at an equal intensity, the aim of this study was to investigate individual and gender differences in personality between healthy participants and those suffering from severe feature MDD.

Subjects and methods: The sample consisted of 632 participants: 385 in the healthy control group and 247 MDD, the latter comprised of patients in their first diagnosed episode or recurrent. The Hamilton Depression Rating Scale (HAM-D) was used to measure symptom severity. Beck's Depression Inventory was administered when depression symptoms had lessened, establishing it as minor when filling out the personality questionnaire (NEO-PI-R).

Results: The results indicate a broad difference in personality between the healthy control and the MDD groups. High neuroticism and low extraversion, accompanied by low scores on openness and conscientiousness, were the most important personality dimensions in understanding distinctions. While agreeableness did not indicate any important role, it did significantly influence the understanding of gender differences within groups. Females were found more agreeable in both groups, but those from the healthy group were also more open and conscientiousness than healthy males. Females from the MDD group were found to be also higher on neuroticism than males of the same group.

Conclusions: A general conclusion from the study is that personality dimensions are more important in understanding vulnerability to depression in comparison to gender differences in personality within groups. As females in the MDD group tend to self-report higher levels of agreeableness and neuroticism than do males in the same group when the level of their depression is categorized as equal MDD-severe type, this may influence practitioners to unequally diagnose depression in males and females.

Key words: gender - personality - depression - MDD

\section{INTRODUCTION}

Depression is a widespread disorder affecting individuals from all walks of life. Those suffering from depression are at a higher risk of also suffering from a broad spectrum of other dysfunctionalities that affect most areas of their personal and public lives (Judd et al. 2000). Although depression is caused by the interaction of multiple factors, many studies have indicated that basic personality dimensions are one of the most crucial factors in being vulnerable to depression (Koorevaar et al. 2013, Kotov et al. 2010, Santor et al. 1997). Another well-known variable strongly related to depression is gender - females have 1.5- to 3-fold increased risk of the onset of MDD (American Psychiatric Association 2013). As gender is a constant in the distribution of depression, it frequently leads to some authors treating depression as a "female disorder". On the other hand a recent meta-analysis underscores the diagnosis of depression as influenced by gender biases (Salk et al. 2017). Their study was conducted on a representative sample of more than 1.7 million females and males. It estimated a small effect size between females and males in depression symptoms and MDD. Thereby, it supports the interpretation of MDD as a female-stereotype disorder, which could be harmful to both females and males. It risks to overdiagnosing depression in females and potentially overmedication; for males, their depression could be overlooked.

Due to the important and recognized role of personality and gender to explain of depression, gender differences on basic personality structure between depressed and non-depressed participants will be explored in this study, as well as any interactions of gender, depression, mental health and personality dimensions. The last is decisive in examining whether gender differences in personality dimensions remain the same for healthy participants and for patients suffering from MDD, as well as to explore whether males differ from females in their self-reports of their personality when the level of their depression is diagnosed as equal. The findings will help in the process of psychodiagnosis in crucially noting differences of personality between genders in MDD that might influence some practitioners to possible overdiagnoses depression in females. 


\section{Personality dimensions and depression}

The five-factor model (FFM) of personality represents the five broad basic personality dimensions that are fundamental and comprehensive in explaining differences between individuals and their behavior. The five personality dimensions remaining relatively stable over one's lifetime are neuroticism, extraversion, openness, agreeableness and conscientiousness (Costa \& McCrae 1992). Thus far, much research has been conducted into exploring the link between the five-factor model of personality and depression.

The factor of neuroticism presents a personal basis that allows for negative affects to become more clearly and quickly felt. In this regard, high neuroticism has been uniformly found to be related to depression (Hill et al. 2011, Noteboom et al. 2016). Goodwin and Gotlib (2004) found that even though females are associated with an increased likelihood of developing MDD, neuroticism has a significant independent contribution in explaining depression apart from gender. A meta-analysis has indicated that those with MDD scored higher on neuroticism but lower on extraversion and conscientiousness than controls (Kotov et al. 2010). It was found that the presence of depression and depression severity were connected to higher scores of neuroticism as well as lower scores on extraversion and consciousness (Koorevaar et al. 2013). In a recent study of NaragonGainey and Simms (2017) empirical support was established for the hypothesis that no single personality dimension is relevant to explaining depression; rather, the three-way interaction of neuroticism, extraversion and conscientiousness.

Openness is the most disagreed upon factor as no clearly established argument as to how much it is linked to depression is present within the literature. Some studies have found a link between a greater range of openness subscales, such as ideas and fantasy, and some psychotic-like phenomena (schizothipy and schiyophrenia spectrum-disorder symptoms) (Chmielewski et al. 2014, DeYang et al. 2012). It was further found that two facets of openness aesthetic and feelings are associated with depression (Wolfenstein \& Trull 1997). Conversely, some authors have found a positive correlation of openness and psychological well being (Lamers et al. 2012). The same factor is linked to having greater life satisfaction and less cognitive impairment in the elderly (Gregory et al. 2010). The connection between agreeableness and depression remains the least researched. However, the results gathered do suggest that agreeableness does relate to happiness (Steel et al. 2008) and positive mental health (Lamers et al. 2012).

\section{Present study}

The aim of this study was to investigate individual and gender differences in personality between healthy participants and those suffering from MDD. Moreover, another goal was to explore interaction between gender differences, group membership and personality dimensions. The aim thereof is to shed light on which variables generate the greatest differences; specifically, whether there are individual differences in personality between groups (depressed and control) or individual differences between genders.

Another goal is to examine whether differences in personality dimensions exist between males and females when all are diagnosed with severe feature MDD. The practical implications thereof are that the practitioner may become better aware of differences in personality between females and males suffering from MDD so that gender difference in personality may not interfere with depression diagnoses and subsequent treatment.

\section{Procedure}

The study was conducted at the Institute of Mental Health and was approved by its ethical committee. Psychiatric evaluation was conducted upon admission into the Institute when the participants were rated on the psychiatric scale for depression. Those who met the criteria for MDD according to the DSM 5 were included in the study. Self-report measures were initiated thereafter when their symptoms of depression increased to the level where their state of depression had a lower influence on personality traits in order that more valid data may be obtained. Even though the affect was lowered when administering these questionnaires, it had not entirely subsided.

\section{SUBJECTS AND METHODS}

\section{Sample and Procedure}

The sample consists of 632 participants divided into two independent groups: a) 385 in the healthy control group (mean age $=25.52 ; \mathrm{SD}=12.70$; from 16 to 70 ) and b) 247 inpatients (mean age $=43.42 ; \mathrm{SD}=14.11$; from 16 to 72) who were diagnosed with MDD. All the participants have signed their written informed consent and the data obtained have been kept confidential.

The MDD patient group consisted only of patients diagnosed as having their first depressive episode or with recurrent depressive disorder. They were all diagnosed using DSM-V criteria for MDD. The exclusion criteria were MDD in bipolar disorders, psychotic disorders, substance abuse, neurological disorders, and intellectual disability. The Hamilton Depression Rating Scale (HAM-D) (Hamilton 1960, 1967) was used to measure the severity of the symptoms. The intensity of depression across both groups of MDD was classified as severe. The average score on the HAM-D scale was $27.86(\mathrm{SD}=0.39)$, which was assessed upon first examination of the patient. Self-report questionnaires were administered when the symptoms of depression had lessened, which was confirmed by Beck's Depression Inventory. The mean score on $\mathrm{BDI}$ was 14.82 ( $\mathrm{SD}=8.89$; from 0 to 28 ), establishing their depression to be at a mild intensity when filling out the questionnaires. 
The control group was comprised of healthy participants who, at the moment of testing, were functional and who did not report a history of significant psychiatric disorders. The control group consisted of $118(30.6 \%)$ males and $267(69.4 \%)$ females, and the MDD group consisted of 79 (32\%) males and 168 $(68 \%)$ females. The groups initially were matched for sex, later analyses of chi-square showed non-significant results $(0.125 ; \mathrm{p}=0.724)$, indicating that both groups did not differ according to gender.

\section{Instruments}

The Hamilton Depression Rating Scale (HAM-D) was applied to patients already diagnosed with depression as to quantify the severity of their symptoms. HAM-D itself is composed of seventeen questions by which the rater assesses the intensity of each symptom according to a five-level (0 to 4 ) or a three-level ( 0 to 2) scale. The score represents the state of the patient: 0 to 6 indicate neither signs nor symptoms of depression, 7 to 17 , mild depression, and, 18 to 24 , moderate to severe. Scores of 24 or higher mark severe depression (Hamilton 1960, 1967).

The Beck Depression Inventory (BDI; Beck et al. 1998 ) is a 21-question multiple-choice self-report inventory. Each question has four statements. Participants must choose the one that represents the best his/her inner states, reflecting a maximum of two weeks in the past. It is composed of items relating to symptoms of depression such as hopelessness and irritability, guilt or feelings of being punished, suicidal thoughts and behavior, as well as physical symptoms such as fatigue, weight loss and lack of interest in sex. The BDI is widely used as an assessment tool both in a clinical practice and research.

NEO Personality Inventory, Revised (NEO-PI-R; Costa \& McCrae 1992), a 240 item self-report scale, was used to assess personality dimensions as classified by the five factor model of personality: Neuroticism, Extraversion, Openness, Agreeableness and Conscientiousness. Participants were instructed to indicate their level of agreement or disagreement with each statement on a 5-point Likert-type answering format ranging from 0 (strongly disagree) to 4 (strongly agree).

\section{RESULTS}

\section{Descriptive statistics and t-tests for MDD and the control group according to personality dimensions}

Table 1 presents the means and the standard deviations of factors of the NEO-PI-R for the total sample separately for groups, as well as their effect size, t-test and significance. The means for the factors of NEOPI-R of the control group are nearly consistent with their respective means of the national normative sample (for norms, see Knezevic et al. 2004). For the control group, the effect size shows that the results obtained for Neuroticism is the same as for normative $(d=0.01)$. While scores for the factors Extraversion and Openness are slightly higher in our sample $(d=0.47 ; d=0.43)$, this effect size is not significantly large. For Agreeableness and Conscientiousness, the scores are slightly lower compared against the normative sample, almost reaching small effect $(\mathrm{d}=0.34 ; \mathrm{d}=0.31)$. Comparing MDD group scores on NEO-PI-R against the normative sample, Neuroticism showed a strong effect size $(d=1.25)$ wherein that MDD group obtained significantly higher results. For Openness and Agreeableness, the effect size was small $(d=0.18 ; d=0.10)$. Scores of the control group showed a strong effect size for Extraversion and Conscientiousness $(\mathrm{d}=0.74 ; \mathrm{d}=0.81)$ in comparison with the normative sample. On the basis of the effect size, it can be concluded that the MDD group's scores differentiate themselves from those of the normative group more than those of the control group.

According to the t-test, the total mean scores for all factors obtained in the control group on NEO-PI-R significantly differ than scores obtained in the MDD group. As expected, Neuroticism was higher for the MDD sample, whereas scores on Extraversion, Openness and Conscientiousness were lower in comparison with the control group. Surprisingly Agreeableness was higher for MDD as their scores indicate that they tend to be more agreeable in their relationships than the participants in the control group.

After it had been found that all five dimensions of the NEO-PI-R differed between the control group and MDD, it was deemed pertinent to examine their effect size (Cohen's d) to further distinguish these two groups (Table 1).

Table 1. Separately listed means and standard deviations for the total sample according to group, including their effectsize, t-test and significance

\begin{tabular}{lccrrrrrrrr}
\hline & \multicolumn{1}{c}{ Total sample } & \multicolumn{2}{c}{ Control group } & \multicolumn{2}{c}{ MDD } & \multicolumn{1}{c}{} \\
& M & SD & \multicolumn{1}{c}{ M } & \multicolumn{1}{c}{ SD } & \multicolumn{1}{c}{ M } & SD & \multicolumn{1}{c}{ d } & t & df & p \\
\hline Neuroticism & 100.69 & 26.40 & 87.84 & 21.98 & 117.16 & 22.74 & -1.31 & -16.046 & 622 & 0.000 \\
Extraversion & 101.58 & 22.03 & 111.89 & 17.98 & 88.04 & 20.29 & 1.24 & 15.365 & 622 & 0.000 \\
Openness & 112.41 & 19.59 & 117.29 & 17.96 & 105.40 & 20.22 & 0.62 & 7.681 & 622 & 0.000 \\
Agreeableness & 115.65 & 18.52 & 113.84 & 18.66 & 118.58 & 16.11 & 0.27 & -3.265 & 622 & 0.001 \\
Conscientiousness & 114.05 & 21.75 & 118.94 & 20.49 & 107.90 & 21.93 & 0.52 & 6.387 & 622 & 0.000 \\
\hline
\end{tabular}


Sanja Nikolic, Ivana Perunicic Mladenovic, Olivera Vukovic, Jasmina Barišić, Dragan Švrakić \& Srdjan Milovanović:
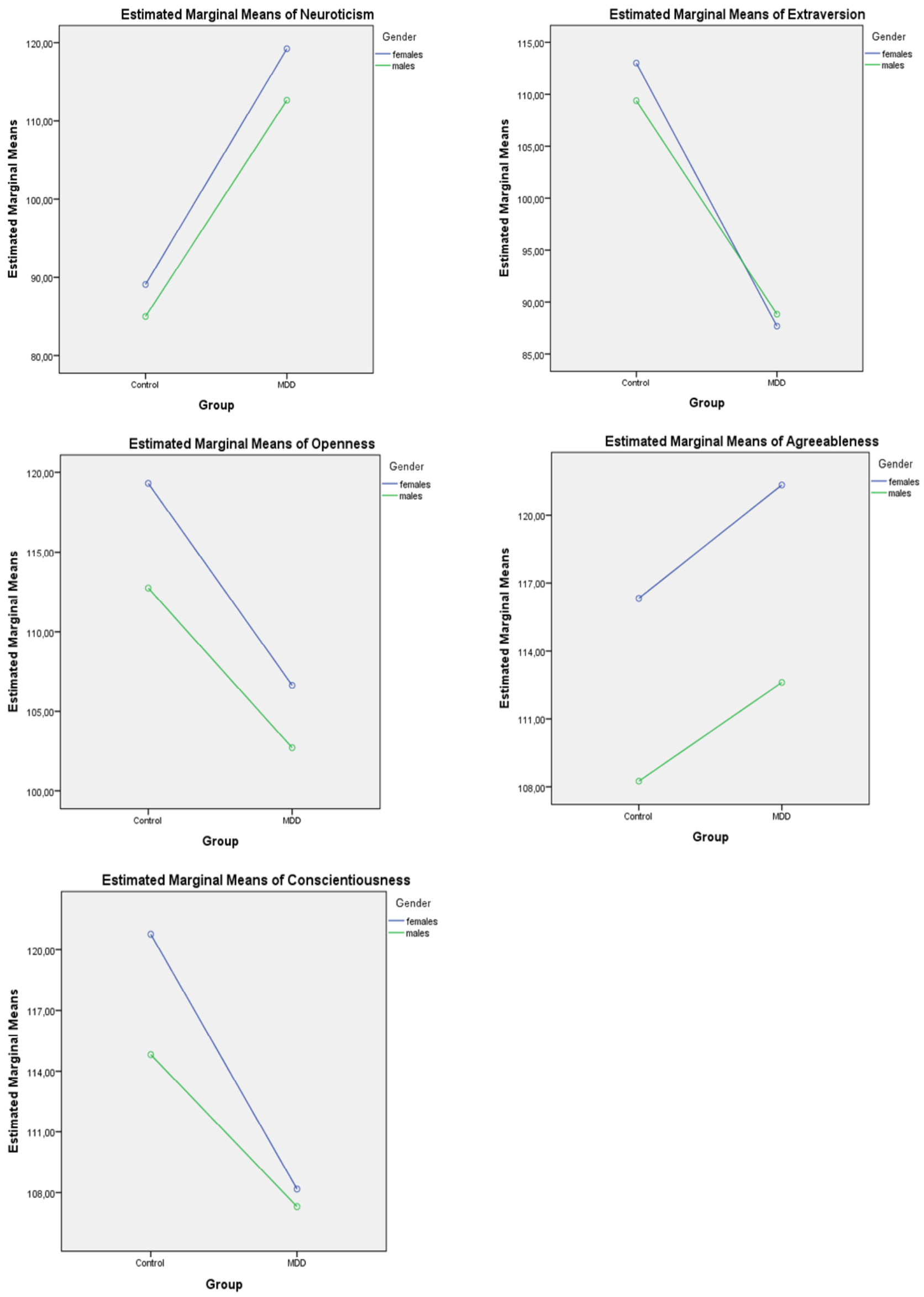

Figure 1. Interactions of gender $\mathrm{X}$ group membership and personality dimensions 
The strongest effect size was for neuroticism, followed by extraversion. These two variables had a large effect size in the two groups of participants. Openness and conscientiousness had a moderate effect size within distinguishing these two groups. The effect of agreeableness distinguishing these two groups is small.

Discriminant Canonical Analyses was applied to differentiate two groups of participantes by analyzing the effect of personality dimensions when the scores of the dimensions were not mutually contaminated by their intercorrelations (Table 2). One significant canonical discriminatory function has been extracted $(\mathrm{r}=0.62 ;$ Wilks` Lambda $=0.621$; Chi-square $=294.98$; $\mathrm{p}<0.001)$. Correlations between personality measures and discriminant function indicate that canonical loading is strongest for neuroticism, then for extraversion, and, finally, for openness and conscientiousness (Table 2). Values of group centroids show that MDD group are higher (0.97) in this function than males $(-0.63)$.

Table 2. Correlations between NEO-PI-R and standardized canonical discriminant functions

\begin{tabular}{lc}
\hline & Function \\
\hline Neuroticism & 0.82 \\
Extraversion & -0.79 \\
Openness & -0.39 \\
Conscientiousness & -0.33 \\
Agreeableness & 0.17 \\
\hline
\end{tabular}

As to whether there were any interactions between gender and group membership (MDD or the control group) and the FFM, the general linear modeling multivariate test indicates no significant interaction (Wilks' Lambda $=0.994 ; \mathrm{F}=0.769 ; \mathrm{p}=0.572$ ). Gender differences behave almost the same for the two groups of participants (Figure 1). When analyzing the effect size in their estimation, it was found that such group differences (as to whether one was depressed or not) create greater distance on personality than gender differences in personality within groups. These results are further confirmed using Cohen's D in estimating effect size (see tables 1, 3, 5).

\section{Personality dimensions by gender in the control group}

The results obtained using a t-test established significant difference between males and females in three personality dimensions. Females in the control group tended to be more open, agreeable and conscientious than males in the same group (Table 3).

Discriminant Canonical Analyses was again applied to differentiate males and females in the control group. One significant canonical discriminatory function has been extracted $(\mathrm{r}=0.35$; Wilks` Lambda $=0.875$; Chisquare $=50.01 ; \mathrm{p}<0.001)$. Correlations between personality measures and discriminant function indicate that canonical loading is strongest for agreeableness, then for openness, and, finally, for conscientiousness, although it does score slightly lower (Table 4). The combination of high scores on agreeableness as the most significant variable in the function and high scores on neuroticism and conscientiousness may be considered as being the most relevant variables in distinguishing males from females in the control group. Values of group centroids show that females are higher $(0.25)$ in this function than males (-0.564).

Table 4. Correlations between NEO-PI-R and standardized canonical discriminant functions in the control group

\begin{tabular}{lc}
\hline & Function \\
\hline Agreeableness & 0.54 \\
Openness & 0.46 \\
Conscientiousness & 0.36 \\
Extraversion & 0.25 \\
Neuroticism & 0.23 \\
\hline
\end{tabular}

\section{Personality dimensions by gender in the MDD group}

The results obtained using the t-test indicates significant differences between males and females in two personality dimensions. Females in the MDD group tend to score higher on agreeableness and neuroticism than males (Table 5).

Table 3. Separately listed means and standard deviations by gender for the control group, including their t-test and significance

\begin{tabular}{lrrrrrrrc}
\hline & \multicolumn{2}{c}{ Males } & \multicolumn{2}{c}{ Females } & & & \\
& M & SD & M & SD & d & t & df & p \\
\hline Neuroticism & 85.00 & 22.10 & 89.10 & 21.84 & 0.19 & 1.682 & 378 & 0.093 \\
Extraversion & 109.38 & 16.66 & 113.00 & 18.46 & 0.21 & 1.817 & 378 & 0.070 \\
Openness & 112.75 & 16.27 & 119.32 & 18.33 & 0.38 & 3.336 & 378 & 0.001 \\
Agreeableness & 108.25 & 18.46 & 116.32 & 18.24 & 0.44 & 3.968 & 378 & 0.000 \\
Conscientiousness & 114.82 & 21.60 & 120.77 & 19.74 & 0.29 & 2.637 & 378 & 0.009 \\
\hline
\end{tabular}


Table 5. Separately listed means and standard deviations by gender for the MDD group, including their t-test and significance

\begin{tabular}{lrrrrrrrr}
\hline & \multicolumn{2}{c}{ Males } & \multicolumn{2}{c}{ Females } & \multicolumn{1}{c}{ t } & df & $\mathrm{p}$ \\
\hline Neuroticism & 112.67 & 23.55 & 119.23 & 22.12 & 0.29 & 2.108 & 242 & 0.036 \\
Extraversion & 88.83 & 20.12 & 87.68 & 20.42 & 0.06 & -0.410 & 242 & 0.682 \\
Openness & 102.71 & 20.05 & 106.64 & 20.24 & 0.19 & 1.413 & 242 & 0.159 \\
Agreeableness & 112.61 & 14.61 & 121.33 & 16.06 & 0.57 & 4.055 & 242 & 0.000 \\
Conscientiousness & 107.31 & 22.01 & 108.17 & 21.96 & 0.04 & 0.285 & 242 & 0.776 \\
\hline
\end{tabular}

Discriminant Canonical Analyses was again applied to differentiate males and females in the MDD group by analyzing the effect of personality dimensions in differing males and females in the MDD sample. One significant canonical discriminatory function has been extracted $(\mathrm{r}=0.31$; Wilks` Lambda $=0.905$; Chi-square $=23.87 ; \mathrm{p}<0.001)$. Correlations between personality measures and discriminant functions show that canonical loading is first strongest for agreeableness and then for Neuroticism. Although this is the case for Neuroticism, it scores slightly lower. The combination of high scores on agreeableness as the most significant variable in the function and high scores on neuroticism may be considered as being the most relevant in distinguishing males from females in the MDD group (Table 6). Values of group centroids show that females are higher (0.22) in this function than males (-0.48).

Table 6. Correlations between NEO-PI-R and standardized canonical discriminant functions in the MDD group

\begin{tabular}{lc} 
& Function \\
\hline Agreeableness & 0.81 \\
Neuroticism & 0.42 \\
Openness & 0.28 \\
Extraversion & -0.08 \\
Conscientiousness & 0.06 \\
\hline
\end{tabular}

\section{DISCUSSION}

The results from the study reveal several findings important in understanding personality dimensions as a component of dispositional factors of vulnerability to MDD. Although mean scores for all FFM personality dimensions were significantly distinct for the MDD group and the healthy control group, further analyses determined that a combination of high neuroticism, low extraversion and (to a lesser extent) low openness and conscientiousness present a personal basis in MDD. Agreeableness indicated no importance in understanding MDD. This result itself aligns with those of previous studies, where a combination of high neuroticism, low extraversion and conscientiousness were found to be substantial for proneness to developing MDD (Naragon-Gainey \& Simms 2017). Although not receiving investigation in their studies, low openness was examined here, thereby adding to their overall results.
Concerning gender, an unforeseen result was found in personality differences between the healthy control and MDD groups. There were larger differences among genders recorded for the healthy participants which followed the control group representing a more heterogeneous population sample. Female participants from the control group tended to be more agreeable, open and conscientious than were males, which disagrees with the finding that females generally score higher on neuroticism (Budaev 1999, Lynn \& Martin 1997). Notwithstanding, this result does concur with others where female participants tended to be more agreeable (Budaev 1999, Goodwin \& Gotilb 2004). A combination of high agreeableness and high neuroticism differ the female from male participants in the MDD group. Consequently, there is a concordance with the results obtained in the study of Budaev (1999) on the student population. It is important to note that the overall level of neuroticism for males and females from the MDD group is higher than for the control. Therefore, albeit males from the MDD group are high on neuroticism, females are significantly higher than their male coparticipants who also scored high on the same dimension.

In both groups, female participants were more agreeable than males. A possible explanation could be found in the content of the domain of agreeableness. Combining submissiveness and love, females generally score higher on this factor (Budaev 1999). From an evolutionary perspective, "women who were more agreeable and nurturing may have promoted the survival of their children and gained an evolutionary advantage" (Costa et al. 2000).

As our study has not found any interaction of gender, group membership and FFM, the results suggest that gender differences on personality are smaller than differences between groups on personality. Such results come in accordance with other well-established findings that gender differences are small relative to individual variations within genders (Costa et al. 2000). Their results were obtained from a total sample of 23,031 participants, stemming from a range of cultures. They concluded that females tend to be higher in negative affect, submissiveness and nurturance, as well as more concerned with feeling than with ideas.

The findings that females from the MDD group tend to report higher levels of neuroticism and agreeableness deserves attention since it reflects the fact that some 
personality characteristics are not the same between males and females, even when the level of depression is constant for both genders (all participants initially were diagnosed as MDD severe type). These results could be further used in diagnosing disorders. They may also help explain the female-stereotype of depression held in psychiatry. In this study, females self-reported a higher tendency to be agreeable as well as to generally feel negative affects - vulnerability, tendencies to anxiety and depression (coming under the domain of neuroticism). While this may lead to them being more easily considered as dependent and insecure (Sulsman \& Page 2004), the result is that females are seen as being more dysfunctional and result in the more frequent diagnosis of depression.

\section{Limitation}

One of the study's strengths is that the personality questionnaire was not administered when the patients were first examined. Rather, it was reserved until when their moment of depression decreased to a mild level or subclinical level. This was done to avoid the state-trait effect which refers to the strong affect otherwise being able to influence personality questionnaires. Regardless of the fact that the study's design lowered this effect by administering the questionnaire approximately two to three weeks after the participants had been diagnosed with MDD and when they started taking antidepressants, the authors are aware that this effect was not totally diminished. However this effect was the same from males and females in the MDD group. Therefore, it may be concluded that this effect did not have any significant influence on the result regarding gender differences in personality in the MDD group.

Self-report measures of personality which are sensitive to different types of strategies in their answering were utilized in the study (Perunicic \& Knezevic 2018). It is unable to be confirmed that the personality dimensions obtained are accurate estimations of the participants' typical behavior. However, it was established that NEO-PI-R could be used as an effective tool in diagnosing depression when aware of gender differences in self-reports for MDD.

\section{CONCLUSION}

Several conclusions may be gathered. Foremost is that the differences in personality between the healthy control and MDD groups are broad. High neuroticism and low extraversion accompanied by the low scores on openness and conscientiousness distinguish the MDD group from its healthy participants.

The factor of agreeableness did not express any important role in understanding the differences between groups. Nevertheless, agreeableness was found to have a significant influence on understanding the gender differences in both participant groups. Whereas female participants tended to be more agreeable in both groups, those from the healthy group also tended to be more open and conscientious than healthy males, while those from the MDD group scored higher on neuroticism than their male counterparts from the MDD group.

No interaction between gender, group membership and personality dimensions were established, indicating that individual differences in personality between groups (being depressed or not) are stronger than within gender variations in personality. However, the obtained gender differences in personality should be kept in mind in order not to underestimate male depression since males self-reported lower levels of neuroticism and agreeableness. In comparison to females, this may be initially viewed as their being more functional and less dependent when their level of depression is equal to that of females. However, this does not indicate that they are indeed suffering from a lower level of depression but might relate to a preheld bias in the diagnosis.

\section{Acknowledgements: None.}

\section{Conflict of interest: None to declare.}

\section{Contribution of individual authors:}

Sanja Nikolic, Ivana Perunicic Mladenovic \& Olivera Vukovic made substantial contribution in acquisition of data and statistical analysis of data;

Jasmina Barisic, Dragana Svrakic \& Srdjan Milovanovic made substantial contribution in interpretation;

Authors participated in drafting the article or revising it critically for important intellectual content.

\section{References}

1. American Psychiatric Association: Diagnostic and statistical manual of mental disorders (5th ed.). Arlington, VA: American Psychiatric Publishing, 2013

2. Beck AT, Ward CH, Mendelson M, Mock $J$ \& Erbaugh $J$ : An inventory for measuring depression. Archives of General Psychiatry 1961; 4:561-71

3. Budaev SV: Sex differences in the Big Five personality factors: Testing an evolutionary hypothesis. Personality and Individual Differences 1999; 26:801-813

4. Chmielewski M, Bagby RM, Markon K, Ring AJ \& Ryder $A G$ : Openness to Experience, Intellect, Schizotypal Personality Disorder, and Psychoticism: Resolving the Controversy. Journal of Personality Disorders 2014; 28:483-499

5. Costa PT \& McCrae RR: Revised NEO Personalyti Inventory (NEO PI-R) and NEO Five-Factor Inventory (NEOFFI) Professional Manual. Psychological Assesment Resource 1992; 36:587-596

6. Costa PT, Terracciano A \& McCrae RR: Gender differences in personality traits across cultures: Robust and surprising findings. Journal of Personality and Social Psychology 2001; 81:322-331 
7. DeYoung CG, Grazioplene RG \& Peterson JB: From madness to genius: The Openness/Intellect trait domain as a paradoxical simplex. Journal of Research in Personality 2012; 46:63-78

8. Goodwin RD \& Gotlib IH: Gender differences in depression: The role of personality factors. Psychiatry Research 2004; 126:135-142

9. Gregory $T$, Nettelbeck $T \&$ Wilson C: Openness to experience, intelligence, and successful ageing. Personality and Individual Differences 2010; 48:895-899

10. Judd LL, Akiskal HS, Zeller PJ, Paulus M, Leon AC, Maser JD \& Keller MB: Psychosocial disability during the long-term course of unipolar major depressive disorder. Archives of General Psychiatry 2000; 57:375-380

11. Hamilton M: A rating scale for depression. Journal of Neurology, Neurosurgery, and Psychiatry 1960; 23:56-62

12. Hamilton M: Development of a Rating Scale for Primary Depressive Illness. British Journal of Social and Clinical Psychology 1967; 6:278-296

13. Knezevic G, Dzamonja-Ignjatovic $T \&$ Đuric-Jocic D: Five-factor personality model [Petofaktorski model licnosti]. Belgrade, RS: Center of Applied Psychology, 2004

14. Koorevaar AML, Comijs HC, Dhondt ADF, Van Marwijk HWJ, Van Der Mast RC, Naarding P \& Stek ML: Big Five personality and depression diagnosis, severity and age of onset in older adults. Journal of Affective Disorders 2013; 151:178-185

15. Kotov R, Gamez W, Schmidt F \& Watson D: Linking "Big" personality traits to anxiety, depressive, and substance use disorders: A meta-analysis. Psychological Bulletin 2010; 136:768-821
16. Lamers SMA, Westerhof GJ, Kovács $V$ \& Bohlmeijer ET: Differential relationships in the association of the Big Five personality traits with positive mental health and psychopathology. Journal of Research in Personality 2012; 46:517-524

17. Naragon-Gainey $K \&$ Simms LJ: Three-way interaction of neuroticism, extraversion, and conscientiousness in the internalizing disorders: Evidence of disorder specificity in a psychiatric sample. Journal of Research in Personality 2017; 70:16-26

18. Noteboom A, Beekman ATF, Vogelzangs $N \&$ Penninx BWJH: Personality and social support as predictors of first and recurrent episodes of depression. Journal of Affective Disorders 2016; 190:156-161

19. Perunicic Mladenovic I \& Knezevic G: Faking amoralism: An ability elusive to both measures of substance and style. Personality and Individual Differences 2018; 120:95-101

20. Salk RH, Hyde JS \& Abramson LY: Gender differences in depression in representative national samples: Metaanalyses of diagnoses and symptoms. Psychological Bulletin 2017; 143:783-822

21. Steel P, Schmidt J \& Shultz J: Refining the Relationship Between Personality and Subjective Well-Being. Psychological Bulletin 2008; 134:138-161

22. Wolfenstein $M \&$ Trull TJ: Depression and openness to experience. Journal of Personality Assessment 1997; 69:614-632

23. Zajenkowski $M$ \& Matthews G: Intellect and openness differentially predict affect: Perceived and objective cognitive ability contexts. Personality and Individual Differences 2019; 137:1-8

Correspondence:

Sanja Nikolic, MD, PhD

Institute of Mental Health

10000 Belgrade, Serbia

E-mail:nikoli.sanja@yahoo.com 